\title{
Accesibilidad física en el transporte para personas con discapacidad y el accionar del Poder Judicial en Argentina. Una mirada desde la rendición de cuentas horizontal*
}

\section{Sergio Hernán Blogna Tistuzza**}

\begin{abstract}
RESUMEN
En sus estudios sobre América Latina y otras regiones, Guillermo O'Donnell encuentra un tipo especifico de democracias políticas, denominadas delegativas, que, lamentablemente, "exhiben un accountability horizontal débil o intermitente". En concreto, ello se demuestra en la existencia de organismos estatales que no cumplen correctamente con su función de controlar y sancionar el accionar de otros organismos. En este trabajo, se analizará el accionar concreto de una de las instituciones de balance: el Poder Judicial en Argentina. Se estudiarán tres diferentes casos referidos al derecho a la accesibilidad en el transporte ferroviario de pasajeros. Estos fallos judiciales y sus consecuencias nos permitirán indagar sobre el nivel de cumplimiento del derecho a la accesibilidad, que impacta en mayor medida a la población con discapacidad, pero también nos aportarán información sobre el rol de contralor de la justicia en este pais, y, en consecuencia, de la calidad de su democracia.
\end{abstract}

\section{PALABRAS CLAVE}

Democracia, Argentina, accountability, discapacidad, politicas públicas.

\begin{abstract}
In studies on Latin America and other regions, Guillermo O'Donnell finds a specific type of political Democracy, called Delegative Democracy, which, unfortunately, has "exhibit weak or intermittent horizontal accountability." In concrete, as it has been demonstrated by the existence of state agencies that do not correctly fulfill their function of controlling and sanctioning the actions of other organizations. In this work, the concrete actions of one of the institutions that offers balance will be analyzed: The Judicial Power in Argentina. Three different cases in reference to the Law of Accessibility in passenger rail transport will be studied. These judicial decisions and their consequences will allow us to investigate the level of compliance with the Law of Accessibility, which has a greater impact on the population with disabilities, but will also provide us with information on the role of controller of justice in this country, and, consequently, of the quality of their democracy.
\end{abstract}

\section{KEYWORDS}

Democracy, Argentina, accountability, disability, public policies.

\footnotetext{
*Artículo recibido el 29 de julio de 2017 y aceptado para su publicación el 4 de diciembre de 2017

** Observatorio de la Discapacidad, Universidad Nacional de Quilmes (sergio_blogna@hotmail.com) ORCID: 00000002-0874-697X
} 
SUM ARIO

1. Introducción

2. Colectivo con discapacidad en Argentina. Justificación de la elección temática

3. Accesibilidad en el transporte ferroviario. Una política pública concreta

4. El rol de la justicia

5. Nivel de accesibilidad física en el transporte ferroviario

6. Conclusiones

\section{INTRODUCCIÓN}

Como señala Mikel Barreda, hay aproximaciones conceptuales heterogéneas sobre la democracia, que pueden organizarse en dos grandes grupos. ${ }^{1}$ En primer lugar, existen aquellas basadas en definiciones procedimentales de democracia, en particular la de poliarquía. ${ }^{2}$ Éstas se enfocan en la garantía de los derechos y libertades indispensables de una democracia electoral. ${ }^{3}$ En segundo lugar, están aquellas que amplían el contenido de la democracia con aspectos sustantivos y finalistas, como el desarrollo económico, la justicia social y la igualdad. ${ }^{4}$

Dentro de los estudios con aproximaciones procedimentales, Guillermo 0’Donnell ${ }^{5}$ y Gerardo Munck ${ }^{6}$ amplían la concepción de la democracia como régimen político, al poner especial atención en el control al ejercicio del poder político y, particularmente, la rendición de cuentas o accountability.

En particular, 0'Donnell entiende a las democracias modernas como la síntesis de tres corrientes históricas: democracia, liberalismo y republicanismo. Define el componente democrático como la existencia de rendición de cuentas vertical, en las elecciones; al componente liberal, como "la idea de que existen

\footnotetext{
${ }^{1}$ BarRedA, Mikel, "La calidad de la democracia: Un análisis comparado de América Latina", Política y gobierno, vol. 18, núm. 2, México, p. 10.

${ }^{2}$ Robert Dahl entiende que un régimen democrático debe satisfacer ocho garantías institucionales básicas: libertad de asociación, libertad de expresión, libertad de voto, elegibilidad para el servicio público, derecho de los líderes a competir en busca de apoyo, diversidad de fuentes de información, elecciones libres e imparciales e instituciones que garanticen que la política del gobierno dependa de los votos y demás formas de expresar las preferencias. DAHL, RoBERT, La poliarquía: participación y oposición, Madrid, Tecnos, 2002.

${ }^{3}$ Altman, David y Pérez-Liñan, Anibal, "Assessing the Quality of Democracy: Freedom, Competitiveness and Participation in Eighteen Latin American Countries", Democratization, núm. 9. Mainwaring, Scot y Pérez-Liñan, Anibal, "Regime Legacies and Democratization: Explaining Variance in the Level of Democracy in Latin America, 1978-2004", documento de trabajo 354, Kellogg Institute for International Studies, 2008.

${ }^{4}$ BARREDA, MikeL, "La calidad de la democracia: un análisis comparado de América Latina", Política y gobierno, vol. 18, núm. 2.

${ }^{5}$ O'Donnell, Guillermo, "Why the Rule of Law Matters", Journal of Democracy, vol. 15, núm. 4.

${ }^{6}$ Munck, Gerardo, "Repensando la cuestión democrática: la región andina en el nuevo siglo", Revista de Ciencia Politica, vol. 30, núm. 1.
} 
ciertos derechos que ningún tipo de poder, menos aún el estatal, pueden invadir" y el componente republicano, como "la idea de que el desempeño de responsabilidades públicas como una actividad dignificante que exige cuidadosa sujeción a la Ley y una entrega devota al servicio del bien público". ${ }^{7}$

Para este autor argentino, cada uno de estos tres componentes propone un aspecto fundamental de la democracia política y el Estado constitucional: el llamado Estado de derecho. ${ }^{8}$ En sus estudios sobre América Latina, O’Donnell encuentra un tipo específico de democracias políticas, denominadas delegativas. $^{9}$ Éstas exhiben una rendición de cuentas horizontal (AH) débil o intermitente ${ }^{10} \mathrm{y}$, como consecuencia, sus componentes liberal y republicano son endebles. ${ }^{11}$

¿Qué entendemos por rendición de cuentas horizontal? Este concepto, que puede traducirse como control interpoderes, hace referencia a la existencia de organismos estatales dedicados a prevenir, indemnizar o sancionar acciones $\mathrm{u}$ omisiones presumiblemente ilegales de otros organismos. En palabras propias del politólogo argentino, el control interpoderes se define como la "existencia de instituciones estatales que tienen el derecho y el poder legal de, y están fácticamente dispuestas y capacitadas para, emprender acciones que van desde el contralor rutinario hasta las sanciones legales o el impeachement" respecto a acciones y omisiones de otras instituciones del Estado que pueden, presuntamente, ser calificadas como ilícitas. ${ }^{12}$

Asimismo, 0'Donnell reconoce que no alcanza con la mera existencia de estas instituciones de control y sanción para garantizar su eficacia. Para ello, se deben cumplir dos condiciones. En primer lugar, estas instituciones estatales deben funcionar en conjunto, se requiere de una red de instituciones que incluya, en su cima, un poder judicial comprometido con esa rendición de cuentas. En segundo lugar, tales instituciones deben tener autonomía, es decir, deben tener fronteras

\footnotetext{
7 O'Donnell, Guillermo, Contrapuntos: estudios escogidos sobre autoritarismo y democratización, Buenos Aires, Paidós, 1997, p. 89.

${ }^{8}$ O'Donnell, Guillermo, Contrapuntos: estudios escogidos sobre autoritarismo y democratización, Buenos Aires, Paidós, 1997, p. 91,

${ }^{9}$ El concepto de democracia delegativa fue acuñado por O'Donnell para referirse a países en donde se celebran elecciones libres y limpias, pero en los cuales los gobernantes se sienten autorizados a actuar sin ser sometidos a las restricciones y los controles de otras instituciones constitucionales, como el Parlamento y el Poder Judicial, ni de diversos organismos estatales o sociales de control. O'Donnell, Guillermo, "Democracia delegativa", Journal of Democracy en español, núm. 1.

${ }^{10}$ O'Donnell, Guillermo, Disonancias. Críticas a la democracia, Buenos Aires, Paidós, 2007, p. 86.

11 O'Donnell, Guillermo, Disonancias. Críticas a la democracia, Buenos Aires, Paidós, 2007, p. 88.

12 O'Donnell, Guillermo, Disonancias. Críticas a la democracia, Buenos Aires, Paidós, 2007, p. 99.
} 
reconocidas y respetadas por los actores relevantes y ciertos "actores deben poder defender las fronteras y recomponerlas cuando sean transgredidas". ${ }^{13}$

El débil funcionamiento del control interpoderes que 0'Donnell detecta en América Latina en general, y en Argentina en particular, entra en contradicción con los diferentes estudios cuantitativos sobre la calidad democrática en este país. Por ejemplo, en el análisis que realiza Mike Barrera sobre los países de la región, concluye que países como Argentina, Chile, Costa Rica, Uruguay y Brasil alcanzan el nivel de calidad de accountability más alto. ${ }^{14}$ Tanto en la variante horizontal como en la vertical, las puntuaciones de estos países están por encima del promedio regional y coinciden con los resultados obtenidos en otros estudios. ${ }^{15}$

Es importante considerar que estos estudios abordan una visión procedimental dahliana, pero más amplia. El estudio realizado por Barreda es uno de los más completos. Considera siete indicadores, de los cuales tres corresponden al desempeño de los mecanismos de rendición de cuentas horizontal, a saber: el índice Political Constraints (v), ${ }^{16}$ el Executive Constraints ${ }^{17}$ y el indicador Estado de derecho del Banco Mundial. ${ }^{18}$

Esta tensión entre los estudios cuantitativos y el análisis que ha realizado Guillermo 0’Donnell se pone en cuestión en este breve trabajo, un primer avance de mi tesis doctoral, ${ }^{19}$ donde analizo la implementación de políticas públicas destinadas al colectivo con discapacidad, así como el funcionamiento de las instituciones de control interpoderes que deben supervisar su implementación.

En términos generales, este artículo se propone analizar la política pública concreta para dar respuesta a la falta de accesibilidad en el transporte ferroviario del Área Metropolitana de Buenos Aires ${ }^{20}$ y el rol que ha tenido una de las tres

\footnotetext{
${ }^{13}$ O'Donnell, Guillermo, Disonancias. Críticas a la democracia, Buenos Aires, Paidós, 2007, p. 102.

${ }^{14}$ BarRedA, MikeL, "La calidad de la accountability de las democracias latinoamericanas", Politai, vol. 2, núm. 2, pp. 18-19.

${ }^{15}$ Alćántara, Manuel, "Luces y sombras de la calidad de la democracia de América Latina", Revista de Derecho Electoral, núm. 6, pp. 1-15. Altman, David y Pérez-Liñan, Anibal, "Assessing the Quality of Democracy: Freedom, Competitiveness and Participation in Eighteen Latin American Countries", Democratization, núm. 9, pp. 85-100. LevinE, DanieL y MoLINA, JosÉ, "La calidad de la democracia en América Latina: una visión comparada", América Latina Hoy, núm. 45, pp. $17-46$.

${ }^{16}$ Mide el desempeño de los actores estratégicos con poder de veto respecto de una política gubernamental (partidos, grupos de legisladores, etcétera).

${ }^{17}$ Mide la extensión de constricciones institucionalizadas al poder ejecutivo (por ejemplo, si el legislativo bloquea las decisiones del ejecutivo o el cambio de los límites constitucionales al ejecutivo por parte de éste).

${ }^{18}$ Informa del grado de vigencia de un sistema legal que establece limites y controles a la acción de los poderes públicos.

${ }^{19}$ Este artículo presenta parte de mi tesis doctoral en la Universidad Nacional de Quilmes (Argentina), bajo la dirección de las doctoras Ana María Mustapic (Universidad Torcuato Di Tella) y Andrea Pérez (Universidad Nacional de Quilmes).

${ }^{20}$ La organización federal de la República Argentina se expresa en la distribución de competencias y funciones en
} 
agencias de balance: el Poder Judicial. ${ }^{21}$ Estudiar cómo el funcionamiento de la rendición de cuentas horizontal incide en la vida cotidiana de los ciudadanos permite aportar una nueva mirada sobre el funcionamiento de nuestra democracia y constatar si en las políticas analizadas se confirma el adecuado funcionamiento institucional o si, por el contrario, se encuentran limitaciones en el control interpoderes.

El presente trabajo está organizado en cinco secciones. En la sección 1, se explica por qué se elige la discapacidad, y el derecho a la accesibilidad física en particular, como temática de análisis. En la sección 2, se explora el marco legal vigente respecto de la accesibilidad física en sistema ferroviario a nivel nacional en Argentina y se presenta la política pública diseñada al respecto. En la sección 3, se indica cómo está organizado el Poder Judicial en Argentina para, luego, explicar tres casos judiciales donde se denuncia la falta de accesibilidad en el transporte. De esta manera, se pretende evaluar qué acción ha tenido la justicia en cada uno de estos casos. En la sección 4, se recurre a distintas fuentes, como informes de la Auditoría General de la Nación, documentos de la Organización de los Estados Americanos (oEA) y del Comité de la Convención Internacional de Discapacidad y expedientes públicos del Gobierno Nacional, con el objetivo de conocer el nivel de cumplimiento de las sentencias judiciales trabajadas.

\section{Colectivo con discapacidad en Argentina. Justificación de la elección TEMÁTICA}

De las diferentes problemáticas que aborda el Estado argentino, hemos decido seleccionar la temática de la discapacidad, y en particular el derecho a la accesibilidad en el transporte ferroviario. Ello se debe a diversas razones. En primer lugar, es importante tener en cuenta que 12,9 \% de la población de Argentina tiene algún tipo de discapacidad (más de cinco millones de personas) $\mathrm{y}$, de este universo, $40 \%$ (dos millones) tiene limitaciones motoras de zona inferior, es decir, dificultades para desplazarse. ${ }^{22}$

\footnotetext{
tres niveles de gobierno (nacional, provincial y municipal). Debido a que el transporte ferroviario del AmBA conecta a la provincia de Buenos Aires con la Ciudad Autónoma de Buenos Aires, éste se encuentra bajo jurisdicción de las autoridades nacionales.

${ }^{21}$ Todas las instituciones que cumplen una función de control interpoderes se pueden organizar en dos grandes grupos: las de balance, que abarcan los tres grandes poderes clásicos del Estado (ejecutivo, el legislativo y judicial); y las agencias con mandato específico, como las auditorias, el ombudsman, las contralorias, fiscalias y semejantes, que fueron creadas recientemente para contrarrestar las deficiencias de las agencias de balance.

22 Instituto Nacional de Estadisticas y Censos de Argentina, Censo 2010, unidad de relevamiento: población, tema: Limitaciones o dificultades permanentes, Argentina, Instituto Nacional de Estadisticas y Censos de Argentina, 2011.
} 
Si bien este colectivo con discapacidad es heterogéneo, ${ }^{23}$ ha sido sistemáticamente vulnerado en sus derechos. ${ }^{24}$ Ha sufrido procesos históricos y estructurales de desigualdad social y, por ello, exigen al Estado que abandone la neutralidad y que realice una acción de protección especial para ellos. En palabras de Víctor Abramovich, grupos como el de personas con discapacidad "demandan un Estado que reconozca la subordinación que sufren frente a otros grupos y, por lo tanto, le exigen que tome partido en su defensa y protección". ${ }^{25}$

Por lo anterior, las personas pertenecientes a este grupo exigen acciones afirmativas que les garanticen ciertos derechos indispensables para asegurar un piso común de ciudadanía. En ese sentido, nuestro país ha adoptado diferentes normas internacionales, regionales y nacionales con el fin de reconocer y trabajar sobre esa desigualdad estructural que sufre el colectivo con discapacidad.

Abordar la problemática que atraviesa este colectivo permite poner en discusión la propia concepción que tenemos sobre la discapacidad. Desde la puesta en vigencia de la Convención Internacional sobre los Derechos de las Personas con Discapacidad, se inició un proceso de cambio en la conceptualización de discapacidad, del modelo médico al modelo social.

Este nuevo paradigma entiende que las causas de la discapacidad no son ni religiosas, ni científicas, sino preponderantemente sociales. Además, considera que las personas con discapacidad pueden aportar a las necesidades de la comunidad en igual medida que el resto - de personas sin discapacidad-. Esto desde la valoración y el respeto de su condición de personas, en ciertos aspectos, diferentes. ${ }^{26}$

Por otra parte, abordar la accesibilidad es una puerta de entrada que permite ejercer otro conjunto de derechos y vivir una vida autónoma. La supresión de barreras es, precisamente, lo que se conoce como accesibilidad, entendida como "la posibilidad de las personas con movilidad reducida de gozar de las adecuadas condiciones de seguridad y autonomía como elemento primordial

${ }^{23}$ El colectivo de la discapacidad está conformado por un grupo social heterogéneo, con personas de distintas clases sociales, nivel educativo y tipología de discapacidad (visual, auditiva, motora, mental). Los distintos términos que se han utilizado (y aún se utilizan), la legislación (como "minusválidos", "personas con discapacidad", "colectivo con discapacidad", "discapacitados", "personas con capacidades diferentes") pretende darle homogeneidad a esta diversidad y darles un tratamiento común.

${ }^{24}$ Samaniego, Pilar, Aproximación a la realidad de las personas con discapacidad en Latinoamérica, Madrid, Cermi, 2006, p. 28.

${ }^{25}$ Abramovich, Victor, "La situación de los derechos humanos en América Latina", Panel Derechos Humanos, Autoritarismo y Democracia. Los aprendizajes de la lucha por los derechos humanos para intervenir en los problemas del presente, Buenos Aires, 18 de mayo de 2009.

${ }^{26}$ Palacios, Agustina, La discapacidad como una cuestión de derechos humanos. Una aproximación a la Convención Internacional sobre los Derechos de las Personas con Discapacidad, Madrid, Cinca, 2007, p. 19. 
para el desarrollo de las actividades de la vida diaria sin restricciones derivadas del ámbito físico urbano, arquitectónico o del transporte, para su integración y equiparación de oportunidades". ${ }^{27}$

De acuerdo a un informe del Comité de oEA dedicado a la discapacidad, sin transporte accesible, ninguna persona "se puede educar, ni atender su salud ni rehabilitar ni tener acceso a la cultura, al deporte o la recreación social. Naturalmente, también se dificulta la inclusión de las personas con discapacidad en situación de pobreza". ${ }^{28}$ Por ello, abordar la falta de accesibilidad es abordar el acceso al conjunto de derechos humanos del colectivo con discapacidad, el cual ha atravesado procesos históricos y estructurales de desigualdad social.

A continuación, nos proponemos explorar en la normativa argentina si se reconoce el derecho a la accesibilidad en el transporte y si existe alguna política pública concreta que la garantice.

\section{AcCESIBILIDAD EN El tRANSPORTE FERROVIARIO. UNA POLITICA PÚBLICA CONCRETA}

El Área Metropolitana de Buenos Aires (AMBA) es la región de mayor concentración demográfica en Argentina. Esta zona del país comprende geográficamente a la Ciudad de Buenos Aires y a los 24 partidos o municipios del conurbano bonaerense, registra una población de alrededor de 13 millones de personas (32 \% del país, según el Censo 2010). Posee una red de transporte ferroviario de pasajeros compuesta por siete líneas de trenes suburbanos, con 218 estaciones, y por seis líneas de subterráneos, con 86 estaciones, conjunto que opera alrededor de 80 kilómetros de vías.

El ferroviario, como todo el sistema de transporte, debe cumplir la normativa nacional e internacional que asegura el derecho a la accesibilidad física. A continuación, vamos a identificar brevemente el marco legal de las acciones que el Estado ha decidido llevar adelante para ello.

En primer lugar, existen dos tratados internacionales específicos sobre los derechos humanos de las personas con discapacidad (la Convención interamericana para la eliminación de todas las formas de discriminación ${ }^{29}$ y la

\footnotetext{
${ }^{27}$ Definición de la Ley 24.314, Sistema de Protección Integral de los Discapacitados, Boletín Oficial de la República Argentina, 8 de abril, 1994.

${ }^{28}$ CEDDIS, "Informe de la República Argentina en virtud del artículo VI.3 de la Convención Interamericana para la eliminación de todas las formas de discriminación contra las personas con discapacidad", 28 febrero, Panamá, 2007, p. 60. [Consulta: 19 de mayo, 2017]. Disponible en: http://www.grupodemujeres.org.ar/descargas/vih-prevenir/DISCAPACIDAD\%20-020CEDDIS_doc_8-07_argentina_esp.doc

${ }^{29}$ Convención interamericana para la eliminación de todas las formas de discriminación contra las personas con discapacidad, adoptada en Argentina por Ley 25.280, Boletín Oficial de la República Argentina, 4 de agosto, 2000.
} 
Convención sobre los derechos de las personas con discapacidad ${ }^{30}$ ). Ambas convenciones establecen principios generales y orientan las acciones que deben llevar a adelante los Estados parte.

Por ejemplo, la Convención interamericana para la eliminación de todas las formas de discriminación contra las personas con discapacidad, en el apartado primero de su artículo tercero, compromete a distintos países a adoptar las medidas necesarias para eliminar la discriminación contra las personas con discapacidad y propiciar su plena integración en la sociedad. Esto incluye las medidas necesarias para eliminar "los obstáculos arquitectónicos, de transporte y comunicaciones que existan, con la finalidad de facilitar el acceso y uso para las personas con discapacidad" (artículo 3).

Por su parte, la Convención sobre los derechos de las personas con discapacidad reconoce de una manera integral los derechos de las personas con discapacidad y expresa el nuevo modelo social de la discapacidad. En relación al derecho a la accesibilidad, esta norma obliga a los países a adoptar las medidas pertinentes para "asegurar el acceso de las personas con discapacidad, en igualdad de condiciones con las demás, al entorno físico, el transporte, la información y las comunicaciones, incluidos los sistemas y las tecnologías de la información y las comunicaciones, y a otros servicios e instalaciones abiertos al público o de uso público, tanto en zonas urbanas como rurales" (artículo 9).

Respecto a la normativa nacional, la Constitución Nacional Argentina le atribuye al Poder Legislativo la facultad de "legislar y promover medidas de acción positiva que garanticen la igualdad real de oportunidades y de trato, y el pleno goce y ejercicio de los derechos reconocidos por esta Constitución y por los tratados internacionales vigentes sobre derechos humanos, en particular respecto de los niños, las mujeres, los ancianos y las personas con discapacidad" (artículo 75, inciso 23).

Dicha prerrogativa se ha plasmado en distintas leyes, como la Ley $22.431,{ }^{31}$ la cual reconoce la discriminación y exclusión que sufren las personas con discapacidad como problema público. En consecuencia, se definen distintas medidas para darles una mejor calidad de vida (política pública). En concreto, en sus artículos 20, 21 y 22, se establecen los criterios de accesibilidad del medio físico, a través de la supresión de las barreras arquitectónicas en el acceso y utilización de todos los medios de transporte público.

\footnotetext{
${ }^{30}$ Convención de los Derechos de las Personas con Discapacidad, adoptada en Argentina por Ley 26.378, Boletín Oficial de la República Argentina, 9 de junio, 2008.

${ }^{31}$ Ley 22.431, Sistema de protección integral de discapacitados, Boletín Oficial de la República Argentina, 20 de marzo, 1981.
} 
Posteriormente, el Decreto reglamentario 914/199732 regula, con precisión, las condiciones y requisitos de accesibilidad al que debería ajustarse todo el transporte ferroviario, "en un plazo no superior a tres años para que el servicio pueda ser utilizado por personas con movilidad y comunicación reducidas especialmente para los usuarios en sillas de ruedas-" (artículo 22).

Si bien no es objetivo de este trabajo profundizar en estas cuestiones técnicas que hacen material la accesibilidad, resulta imprescindible presentar algunos criterios básicos. Principalmente, la normativa establece la necesidad de instalar elementos arquitectónicos o de mobiliario urbano que permitan eliminar las barreras con las que se encuentran las personas con discapacidad a la hora de utilizar los transportes ferroviarios.

Por ejemplo, se requieren rampas, ascensores o medios de elevación alternativos para el acceso a las estaciones; la instalación de apoyos isquiáticos ${ }^{33} \mathrm{y}$ solados hápticos ${ }^{34}$ en los andenes, así como la habilitación de baños accesibles para personas con discapacidad en las estaciones.

Ahora bien, debemos tener presente que una vez diseñada una política pública, plasmada en la normativa identificada, es necesaria su correcta implementación para dar respuesta al problema público que se quiere atender (las barreras arquitectónicas en el trasporte). ${ }^{35}$ En caso de que esta política pública no se haya implementado o no haya alcanzado sus objetivos (eliminar las barreras), se estarán vulnerando los derechos del colectivo con discapacidad.

Una forma de indagar en ello es a través del Poder Judicial, una de las agencias de balance del control interpoderes. Entonces se puede explorar si la justicia ha recibido denuncias por la vulneración de este derecho y, así, analizar su accionar y rol en ellas. Esto nos permitirá conocer si existe un acceso real y efectivo de las personas con discapacidad al transporte ferroviario en igualdad de condiciones.

\footnotetext{
32 Decreto 914, Reglamentación de los artículos 20, 21 y 22 de la Ley 22.431, Boletín Oficial de la República Argentina, 18 de septiembre, 1997.

${ }_{33}^{33}$ Son elementos que permiten el descanso de las personas sin necesidad de tener que sentarse e incorporarse.

${ }^{34}$ Los solados hápticos son señalizadores para personas de baja visión o nula. Se colocan en el piso para señalar al peatón, a través de su textura, que está ante la presencia de un peligro o ante un obstáculo como escaleras o bordes peligrosos. Existen de tres tipos: los direccionales o guia, que señalan hacia dónde ir; los de prevención, que avisan de la cercanía con zonas de riesgo; y los de peligro, que alertan sobre la posibilidad de caída ante la llegada a los bordes.

${ }^{35}$ Tamayo Sáez, Manuel, "El análisis de las políticas públicas", en Rafael Bañón, Ernesto Carrillo (comps.), La nueva administración pública, Madrid, Alianza, 1997, p. 2.
} 


\section{El ROL DE LA JUSTICIA}

El sistema de justicia de la República Argentina está compuesto por el Poder Judicial de la Nación y el Poder Judicial de cada una de las provincias. Integran también el sistema de justicia argentino el Ministerio Público Fiscal, el Ministerio Público de la Defensa y el Consejo de la Magistratura.

La organización judicial responde al carácter federal del Estado Argentino. De este modo, por un lado, existe una Justicia Federal con competencia en todo el país que atiende en materia de estupefacientes, contrabando, evasión fiscal, lavado de dinero, y otros delitos que afectan a la renta y seguridad de la Nación. Por otro lado, cada una de las provincias argentinas cuenta con una Justicia Provincial -también denominada justicia ordinaria-, para el tratamiento de los delitos comunes, con sus propios órganos judiciales y legislación procesal.

El Poder Judicial de la Nación se encuentra conformado por la Corte Suprema de Justicia de la Nación, el Consejo de la Magistratura de la Nación, los Juzgados de Primera Instancia y las Cámaras de Apelaciones. Este Poder ejerce el control de constitucionalidad, ${ }^{36}$ a través de un sistema difuso. Es decir, corresponde a todos los jueces, sin distinción de categorías, jurisdicción nacional o provincial, ejercer esa función.

En concreto, esto significa que cualquier ciudadano o ciudadana puede denunciar la inconstitucionalidad de una norma o la vulneración de un derecho, ante cualquier tribunal de primera instancia -tribunal federal si la norma es nacional o tribunal provincial si la norma es subnacional-. Todas estas sentencias son apelables ante una segunda instancia, conformada por distintas cámaras federales de apelación según el fuero. Por último, en la cima de la jerarquía, se encuentra la Corte Suprema de Justicia de la Nación, el máximo tribunal de justicia del país.

A través del Sistema Argentino de Información Jurídica ${ }^{37}$ sobre la jurisprudencia de los tribunales nacionales de la Ciudad de Buenos Aires, hemos detectado tres casos en los que se denuncia la falta de accesibilidad en el transporte ferroviario del Área Metropolitana de Buenos Aires. A continuación,

\footnotetext{
${ }^{36}$ El control de constitucionalidad es el mecanismo jurídico por el cual se revisan las normas ordinarias y, en caso de contradecirse con la Constitución, se invalidan las de rango inferior.

${ }^{37}$ Sistema llevado a cabo por Dirección Nacional del Sistema Argentino de Información Jurídica (SAI)), un organismo dependiente de la Secretaría de Planificación Estratégica del Ministerio de Justicia y Derechos Humanos de la Nación. Tiene a su cargo el ordenamiento, sistematización, actualización y edición de las normas jurídicas, la jurisprudencia y la doctrina en Argentina.
} 
expondremos el planteamiento de estos casos, así como su recorrido por las distintas instancias judiciales.

En primer lugar, se encuentra el caso de María Inés Verbrugghe. La línea Mitre es una línea ferroviaria que conecta la Ciudad de Buenos Aires con la zona norte del área metropolitana, a 32 kilómetros. En la década de los noventa, este servicio fue concesionado a la empresa Trenes de Buenos Aires (TBA), con lo cual se inició un proyecto de remodelación integral de estaciones. Éste incluía la instalación de máquinas expendedoras de boletos y molinetes en los andenes para un mayor control del acceso.

Sin embargo, estas innovaciones impusieron nuevas barreras a las personas con movilidad reducida. Por su estructura, tamaño y mecanismo, los molinetes no pueden ser utilizados por ellas, y la altura de las máquinas expendedoras impide que sean accionadas desde una silla de ruedas. Por tanto, resulta imposible activar los comandos desde la silla y no es factible visualizar la pantalla en la que se indican los pasos para la compra de los pasajes.

María Inés Verbrugghe es docente y se encuentra en una situación de discapacidad motora que la obliga a trasladarse en silla de ruedas. Para llegar a su lugar de trabajo, utilizaba habitualmente la línea Mitre, pero con las modificaciones que se implementaron como parte del proyecto de remodelación integral de estaciones, empeoraron las condiciones de accesibilidad. Por ello, en 1998, con el patrocinio del Centro de Estudios Legales y Sociales, presentó una acción de amparo colectivo contra el Estado nacional (Ministerio de Economía, Secretaría de Transporte) y contra la empresa TBA.

En su amparo, denunció que la colocación de molinetes y expendedoras violaba la ley 22.431 y sus modificatorias, de Accesibilidad de Personas con Movilidad Reducida, porque dificultaban aún más la utilización del servicio. Con este amparo, también cuestionaba el rol de la Comisión de Regulación de Trasporte, por su responsabilidad ante la falta de control sobre las obras realizadas por la empresa y por no haber sancionado la colocación de esos obstáculos, prohibidos por la ley.

María Inés Verbrugghe exigía con esta acción judicial que se garantizara el derecho a la igualdad de las personas con discapacidad en el acceso al transporte ferroviario en condiciones de autonomía y seguridad, y reclamaba la adecuación de ese servicio público a la normativa vigente.

En primera y segunda instancia, la justicia hizo lugar a su reclamo, pero los denunciados decidieron apelar la decisión. En septiembre de 2000, la Corte Suprema de Justicia de la Nación confirmó el fallo de Cámara que había condenado a la empresa "a ejecutar las obras necesarias para habilitar en todas las 
estaciones del ramal Mitre accesos alternativos a los molinetes, que permitan a las personas con discapacidad motora o movilidad reducida, acceder al servicio en condiciones igualitarias al resto de los usuarios [...] en un plazo no mayor de 60 días".

También dejó firme la condena al Estado nacional a "fiscalizar la efectiva realización de las obras y la adecuación a la normativa vigente". Lamentablemente, pasaron los meses y la sentencia no se cumplía. La estrategia jurídica había sido un éxito y el precedente judicial devenía en garantizar la accesibilidad en el transporte, para asegurar así la inclusión de las personas con discapacidad. Sin embargo, la empresa no realizó las adecuaciones necesarias, ni el ente de contralor cumplió con su función.

En mayo de 2003, a cinco años de haber iniciado el amparo, la jueza de la causa dictó una resolución por la que ordenó

intimar en forma solidaria a la empresa concesionaria Trenes de Buenos Aires S.A. (тва) y al Estado nacional, Secretaría de Transporte, para que en el plazo de 10 (diez) días de notificada la presente, procedan a habilitar en todas las estaciones del ramal Mitre, accesos alternativos a los molinetes que puedan ser utilizados por las personas con discapacidad motora o movilidad reducida [...] en condiciones de autonomía y seguridad; ello bajo apercibimiento de aplicar, respecto de la empresa Trenes de Buenos Aires, astreintes equivalentes a $\$ 10.000$ por cada día de demora en el cumplimiento de lo aquí dispuesto y respecto del Estado nacional, de considerar a los funcionarios responsables, incursos en el delito previsto en el artículo 239 del Código Penal. ${ }^{38}$

El Gobierno y la empresa recurrieron en queja ante la Cámara de Apelaciones en lo Federal y Contencioso Administrativo Sala 1, la cual confirmó la resolución. Una vez más, тBA presentó un recurso extraordinario ante la Corte Suprema de Justicia de la Nación, pero este intento tampoco le sirvió: el recurso extraordinario fue rechazado y la resolución quedó firme.

\footnotetext{
${ }^{38}$ Las astreintes son condenaciones conminatorias de carácter pecuniario que los jueces pueden aplicar a quien no cumple una obligación que le fuera impuesta en una resolución judicial, estando en condiciones de cumplirla. Perdura la vigencia de la conminación mientras no cese el incumplimiento; puede aumentarse o disminuirse la misma si no resultare eficaz y puede también quedar sin efecto si el incumplidor justificare su actitud.
} 
Días después de esta sentencia, el Poder Ejecutivo firmó la Resolución 27/2003 de la Secretaría de Transportes, ${ }^{39}$ por la cual se instruye a la Comisión Nacional de Regulación del Transporte se proceda

a habilitar en todas las estaciones del ramal Mitre, accesos alternativos a los molinetes que puedan ser utilizados por las personas con discapacidad motora o movilidad reducida, para acceder al servicio en condiciones de autonomía y seguridad (artículo 1), [se determine] las obras y/o acciones a realizar (artículo 2) [y que diariamente se realicen] inspecciones y controles, a efectos de verificar la evolución del cumplimiento de las acciones establecidas en los Artículos 1 y 2 de la presente Resolución a cargo de la empresa concesionaria de servicio de transporte ferroviario de pasajeros Trenes de Buenos Aires S.A. (artículo 3).

Luego del dictado de esta resolución no hubo nuevos movimientos en el expediente judicial, por lo tanto, no podemos saber si se ha dado cumplimiento a la misma.

El segundo caso corresponde al Defensor del Pueblo de la Nación. En 2006, tres años después de la sentencia de la Corte Suprema argentina en el caso de María Inés Verbrugghe sobre la falta de accesibilidad en el tren Mitre, el Defensor del Pueblo de la Nación presentó una acción de amparo contra Trenes de Buenos Aires S.A., concesionaria a cargo de las líneas Mitre y Sarmiento.

Esta medida judicial solicitaba a la justicia que se garantizara la prestación del servicio de transporte ferroviario a los usuarios de esas líneas en forma digna y eficiente, y que se garantizara el desplazamiento de personas con discapacidades, de acuerdo con lo establecido por el artículo 75, inciso 23 de la Constitución Nacional y la Ley 24.314, de Accesibilidad de Personas con Movilidad Reducida.

La acción también buscaba que el Estado nacional, en particular la Secretaría de Transporte, ejecutara los controles y acciones necesarias para que la codemandada cumpliera debidamente con las obligaciones emergentes del contrato de concesión y que, una vez que se haga lugar al amparo, "extreme los controles necesarios para garantizar el efectivo acatamiento de la sentencia dictada en autos".

El 23 de noviembre de 2006, el juez de primera instancia rechazó la acción de amparo "por no resultar la vía procesal idónea y necesitar de una mayor

\footnotetext{
${ }^{39}$ Resolución 27/2003, Boletín Oficial de la República Argentina, 25 de julio, 2003.
} 
amplitud de prueba y debate". Ante la apelación del defensor del pueblo, la causa fue considerada por la Cámara Nacional de Apelaciones en lo Contencioso Administrativo Federal, la cual confirmó la sentencia y rechazó el amparo el 29 de diciembre de 2009.

La Defensoría del Pueblo presentó un recurso de queja extraordinario ante la Corte Suprema de Justicia, quien el 24 de junio de 2014 revocó la sentencia apelada y remitió el caso al tribunal de origen, a fin de que se dictara un nuevo pronunciamiento para garantizar la accesibilidad. ${ }^{40}$ Luego de este último fallo, no se ha registrado ningún nuevo pronunciamiento, pero sí podemos afirmar que el Máximo Tribunal asume un rol activo en la defensa de los derechos humanos de las personas con discapacidad, como ocurrió en el caso Verbrugghe.

El tercer caso corresponde a Antonieta Aviani, una abogada de 33 años que se traslada en silla de ruedas. Aviani realizó varios reclamos ante la empresa Metrovías S.A., concesionaria del transporte ferroviario subterráneo de la Ciudad de Buenos Aires, por la falta de ascensores en las estaciones y el mal funcionamiento de los instalados.

En 2006, cansada de no obtener respuesta, decidió presentar una acción de amparo contra la empresa y la CNRT, por la omisión en que habían incurrido al no efectivizar su obligación de adecuar todas las estaciones del servicio en cuestión. Lo hizo con el patrocinio de la Asociación por los Derechos Civiles, una oNG dedicada a temas jurídicos e institucionales. ${ }^{41}$

En junio de 2007, el Juzgado Contencioso Administrativo Federal 10 falla a favor de la actora. Ordenó a Metrovías y al Estado nacional que, en el plazo de seis meses, proveyeran todo lo necesario para la elaboración y aprobación de los planes de obras pendientes, destinados a posibilitar el acceso a medios de circulación para permitir el desplazamiento de las personas que utilizan sillas de ruedas. Asimismo, debían fijarse términos razonables de finalización y se exhortó a la CNRT a fiscalizar el cumplimiento de las obligaciones impuestas a Metrovías.

Tanto la empresa Metrovías S.A., como el Estado nacional, decidieron apelar la sentencia. ${ }^{42}$ En octubre de 2008, el caso llegó a la Cámara Nacional de

\footnotetext{
${ }^{40}$ Defensor del Pueblo de la Nación c/ Trenes de Buenos Aires (твA) y otro s/ amparo ley 16.986, Sentencia, Corte Suprema de Justicia de la Nación, Buenos Aires, 24 de junio, 2014.

41 "Obligan a que la red de subte sea accesible para discapacitados", Clarín. [Consulta: 17 de junio, 2017]. Disponible en: http://www.clarin.com/ediciones-anteriores/obligan-red-subte-accesible-discapacitados_0_rksgvhgyAFg.html ${ }^{42}$ A fines del año 2012 se produjo la transferencia del servicio de subterráneos del Estado Nacional a la órbita de la Ciudad de Buenos Aires, en concreto a SBASE (Subterráneos de Buenos Aires Sociedad del Estado), como órgano de aplicación de la ley. Se encargaría del desarrollo y la administración del sistema de infraestructura del transporte subterráneo, su mantenimiento y la gestión de los sistemas de control de la operación del servicio. De esta manera, la CNRT dejó de ser el órgano de control de este servicio, pasando entonces a ser responsabilidad del Ente Único Re-
} 
Apelaciones en lo Contencioso Administrativo Federal, la cual decidió confirmar la sentencia recurrida. En los fundamentos de su decisión, se afirma "que no hay justificativo para que se haya dejado de lado a una parte de nuestros habitantes por carecer de aptitudes plenas debiendo soportar que no se les brinde la ayuda necesaria para superar sus dificultades motrices". ${ }^{43}$

Tanto en primera como en segunda instancia, la justicia argentina sustenta sus decisiones en la gran cantidad de normativa interna e internacional que regulan los derechos humanos en general, y los de las personas con discapacidad en particular. Entre éstos, destacan la Constitución Nacional, en su artículo 16 sobre la igualdad de derechos ante la ley; el artículo 42, sobre la existencia del derecho a un trato equitativo en relación al consumo; el artículo 43, que posibilita accionar contra cualquier forma de discriminación, y el artículo 75, inciso 22, donde se le otorga jerarquía constitucional a los tratados internacionales sobre derechos humanos, y el inciso 23, que establece la igualdad real de oportunidades y de trato, el pleno goce y ejercicio de los derechos reconocidos por la Constitución y por los tratados internacionales vigentes sobre derechos humanos, en particular respecto a personas con discapacidad.

En el plano internacional, están vigentes la Convención sobre los derechos de las personas con discapacidad y su Protocolo facultativo y la Convención Interamericana para la Eliminación de Todas las Formas de Discriminación contra las Personas con Discapacidad, entre otras. La primera fue ratificada por Argentina en el año 2008, y es en ese momento que comienza a ser parte del conjunto normativo al cual debe someterse.

"Es claro que Argentina no cumple de manera absoluta con las disposiciones establecidas en el plexo normativo tanto interno como externo", sostiene el fallo de la Cámara Nacional de Apelaciones en lo Contencioso Administrativo Federal, que luego explica: "Desde el año 1981 se les otorga un plazo a las empresas concesionarias para que pongan a tono el servicio de subterráneo de manera que sea accesible por todos, encontramos la Ley 22.431/81 que le otorgaba un plazo de 10 años para hacer las obras, la Ley 24.314/94 con un plazo de 3 años, el decreto 914/97 que le otorgaba el plazo de 6 meses a 3 años, todas ellas vencidas e incumplidas".

gulador de los Servicios Públicos de la Ciudad de Buenos Aires.

${ }^{43}$ Véase un análisis completo del fallo en Galvagno, Patricia Luján, "Derecho subterráneo", en Actas de II Jornadas Nacionales "Discapacidad y Derechos". [Consulta: 12 de marzo, 2017]. Disponible en: http://www.derecho.uba. ar/academica/centro-desarrollo-docente/documentos/segundas-jornadas-nacionales-discapacidad-y-derechos/ eje-1-legislacion-y-jurisprudencia.pdf 
En síntesis, los jueces decidieron otorgarle a la empresa concesionaria un nuevo plazo para que readecúe las estaciones y cumpla con el plexo normativo descripto. Ante esta decisión, el Estado nacional presentó un recurso de queja ante la Corte Suprema de Justicia de la Nación. Como en el caso Verbrugghe, el 16 de diciembre de 2008, el Máximo Tribunal argentino rechazó el recurso extraordinario y la resolución de primera instancia quedó firme. ${ }^{44}$

Como vimos a lo largo de este apartado, en los tres casos analizados, el Poder Judicial ha cumplido con su función de balance, dando lugar a las denuncias recibidas y ordenando al Gobierno nacional y las empresas el cumplimiento del marco legal vigente. Así, vemos en Argentina un control interpoderes positivo, como lo han señalado los estudios cuantitativos mencionados en la introducción de este trabajo.

Sin embargo, para poder sostener esta afirmación, debemos tener presente que no alcanza con el dictado de sentencias para definir la rendición de cuentas horizontal como positivo sino que, además, estas sentencias deben ser respetadas y cumplidas, es decir, se debe hacer efectivo el acceso al derecho. En la siguiente sección nos proponemos abordar esta cuestión.

\section{NIVEL DE ACCESIBILIDAD FÍSICA EN EL TRANSPORTE FERROVIARIO}

El objetivo del presente apartado es reflexionar sobre el cumplimiento de los tres fallos presentados y, en consecuencia, el acceso al transporte ferroviario de pasajeros en igualdad de condiciones para las personas con discapacidad. Como dentro de los expedientes judiciales no existen informes que den cuenta del cumplimiento de las sentencias, necesitamos trabajar con otras fuentes para poder dar respuesta a nuestras interrogantes.

En este marco, tomaremos en cuenta un documento elaborado por el Comité para la eliminación de todas las formas de discriminación contra las personas con discapacidad (CEDDIS) de la Organización de Estados Americanos (oEA), un informe de la Auditoría General de la Nación (AGN), un expediente propio de la Comisión Nacional de Regulación del Transporte (CNRT), el Informe País del Comité de la Convención de Naciones Unidas y diferentes expedientes, en respuesta a pedidos de información pública al Gobierno Nacional. Esta diversidad de documentos brinda distintas miradas sobre el tema en análisis.

\footnotetext{
${ }^{44}$ Aviani, Antonieta c/ cnRT y otro s/ amparo, Sentencia, Corte Suprema de Justicia de la Nación, Buenos Aires, 16 de diciembre de 2008.
} 
En primer lugar, el informe elaborado por el CEDDIs, en el año 2007, consideró que la accesibilidad en general, y la del transporte en particular, "es una de las mayores dificultades que afrontan las personas con discapacidad en la República Argentina, donde no se ha mantenido una línea permanente de gestión en la materia". ${ }^{45}$

Respecto a las estaciones ferroviarias del AMBA, el Comité encontró que las obras de accesibilidad necesarias fueron estipuladas por la Resolución de la Secretaría de Transporte 94/2004, pero que "la renuencia o la negativa, por parte de las empresas concesionarias [...] han paralizado lo que podría haber sido un avance significativo en materia de accesibilidad" ${ }^{46}$ Esta breve cita, del extenso informe producido por el comité de la OEA, nos ofrece una primera respuesta sobre la falta de accesibilidad en el transporte.

En segundo lugar, la Auditoría General de la Nación ${ }^{47}$ firmó, en 2008, su Actuación 132/08, sobre el "Sistema de Protección Integral de los Discapacitados (SPID), en el transporte público de pasajeros (automotor, ferroviario y subterráneo) que presta servicio en el Área Metropolitana de Buenos Aires”. En este documento, se presenta la situación de cada una de las líneas ferroviarias, con información y fotografías, de forma muy precisa. En sus conclusiones, se afirma que el "sistema ferroviario no es apto para ser utilizado en forma autónoma por personas con capacidades diferentes, especialmente por usuarios que se trasladan en sillas de ruedas". ${ }^{48}$

Para ilustrar esta afirmación, ejemplifiquemos lo que sucede con datos de la línea Urquiza, donde "de las 23 estaciones inspeccionadas, se han obtenido evidencias de que doce, $52.17 \%$, son inaccesibles por diversas razones (ejemplo: falta de rampas con dimensiones reglamentarias para acceder a la zona de ascensores o al andén mismo, falta de ascensores para el acceso a los

\footnotetext{
${ }^{45}$ CEDDIS, "Informe de la República Argentina en virtud del artículo VI.3 de la Convención Interamericana para la eliminación de todas las formas de discriminación contra las personas con discapacidad", 28 febrero, Panamá, 2007, p. 61. [Consulta: 19 de mayo, 2017]. Disponible en: http://www.grupodemujeres.org.ar/descargas/vih-prevenir/DISCAPACIDAD\%20-020CEDDIS_doc_8-07_argentina_esp.doc

${ }^{46}$ CEDDIS, "Informe de la República Argentina en virtud del artículo VI.3 de la Convención Interamericana para la eliminación de todas las formas de discriminación contra las personas con discapacidad", 28 febrero, Panamá, 2007, p. 62. [Consulta: 19 de mayo, 2017]. Disponible en: http://www.grupodemujeres.org.ar/descargas/vih-prevenir/DISCAPACIDAD\%20-020CEDDIS_doc_8-07_argentina_esp.doc

${ }^{47}$ La AGN es un organismo constitucional con autonomía funcional que asiste técnicamente al Congreso de la Nación en el ejercicio del control externo del sector público nacional mediante la realización de auditorías y estudios especiales. ${ }^{48}$ Auditoria General de la Nación, 2008, Actuación Agn 132/08, Buenos Aires, p. 49. [Consulta: 17 de mayo, 2017]. Disponible en: http://www.agn.gov.ar/informes/informesPDF2010/2010_029.pdf
} 
andenes)". ${ }^{49}$ Como vemos, este documento confirma con inspecciones in situ las observaciones planteadas por el cEDDIs.

En tercer lugar, debemos considerar el documento publicado, en 2012, por parte del Comité sobre los Derechos de las Personas con Discapacidad, como parte del proceso de evaluación a Argentina en el cumplimiento de la convención internacional. El mismo ha sido elaborado por distintas organizaciones de la sociedad civil de Argentina ${ }^{50}$-Redi, CELS, FAICA, Fendim, ADC- y aporta una mirada desde las organizaciones de personas con discapacidad sobre el goce de sus derechos.

En concreto, denuncian el incumplimiento de la normativa sobre accesibilidad:

Las políticas de Estado en materia de transporte se encuentran condicionadas: 1. En las grandes ciudades el transporte público está concesionado a empresas privadas. El contralor, así como las sanciones aplicadas por parte de la Comisión Nacional de Regulación del Transporte, órgano autónomo dependiente de la Secretaría de Transporte de la Nación, resultan irrelevantes. Dicho organismo integra el Comité de Asesoramiento y Contralor de la Ley 24.314. Existen aún líneas de ferrocarril a las que es imposible ingresar con silla de ruedas, y alargamiento de recorridos debidos a cierres de puntas de andenes, que resultan demasiado extensos a quienes caminan con dificultad. A su vez, no se implementan en el conjunto de los transportes públicos adecuaciones para PCD auditiva, que aseguren información visual de todo aquello que se anuncia verbalmente. Se constata la persistencia de inaccesibilidad en ferrocarriles y subterráneos, no obstante denuncias y recursos de amparo que datan de 1999 y de 2006 entre muchos otros, con fallo positivo desde la Justicia, pero sin cumplimiento de sentencia hasta la fecha.

248 Este documento aporta una aproximación distinta sobre la temática planteada: la de la voz de las organizaciones que han reclamado al organismo

\footnotetext{
${ }^{49}$ Auditoría General de la Nación, 2008, Actuación Agn 132/08, Buenos Aires, p. 51. [Consulta: 17 de mayo, 2017]. Disponible en: http://www.agn.gov.ar/informes/informesPDF2010/2010_029.pdf

${ }^{50}$ Este informe, conocido como CRPD/C/ARG/1, fue presentado en las sesiones 79 y 80 del Comité de la onu, celebradas el 19 y 20 de septiembre de 2012. [Consulta: 17 de mayo, 2017]. Disponible en: http://www.cels.org.ar/common/ documentos/Informe\%20alternativo-\%201ra\%20evaluaci\%C3\%B3n\%20Argentina-\%20CRPD\%20-\%20FINAL.pdf
} 
internacional. Ésta se encuentra en la misma línea que las fuentes previamente señaladas.

En cuarto y último lugar, consideramos relevante compartir información pública solicitada al Gobierno Nacional y al Gobierno de la Ciudad Autónoma de Buenos Aires, en distintos pedidos entre los años 2013 y 2017. Estos expedientes brindan el resultado de los relevamientos oficiales del sistema de transporte ferroviario (de trenes y de subterráneos) y confirman -de forma oficial- la falta de cumplimiento de la normativa de accesibilidad.

El expediente 110509/2013 de la Comisión Nacional de Regulación del Transporte detalla el resultado de un relevamiento técnico realizado en las siete líneas ferroviarias del Área Metropolitana de Buenos Aires. Debido a la variedad y complejidad de los datos, hemos decidido sistematizarlos y elaborar el cuadro 1. En éste, exponemos la cantidad total de estaciones de cada línea ferroviaria, así como información sobre cuántas de ellas cumplen con los requisitos de accesibilidad dispuestos en la normativa (rampas, baños accesibles, solados hápticos, ascensores y apoyos isquiáticos).

Cuadro 1. Elementos de accesibilidad en las estaciones de las líneas San Martín, Sarmiento, Mitre, Belgrano Sur, Roca y Urquiza, para el año 2013

\begin{tabular}{|l|c|c|c|c|c|c|}
\hline Linea & $\begin{array}{c}\text { Cantidad } \\
\text { estaciones }\end{array}$ & Rampas & $\begin{array}{c}\text { Solados } \\
\text { hápticos }\end{array}$ & Baños & Ascensores & $\begin{array}{c}\text { Apoyos } \\
\text { isquiáticos }\end{array}$ \\
\hline Urquiza & 23 & 10 & 1 & 2 & 9 & no informa \\
\hline Roca & 69 & 6 & 10 & 9 & 0 & 14 \\
\hline Belgrano Sur & 30 & 4 & 16 & 15 & 0 & 0 \\
\hline San Martín & 20 & 0 & 0 & 0 & 0 & 2 \\
\hline Mitre & 37 & 8 & 0 & 0 & 2 & 6 \\
\hline Sarmiento & 17 & 0 & 0 & 0 & 3 & 2 \\
\hline $\begin{array}{l}\text { Belgrano } \\
\text { Norte }\end{array}$ & 22 & 1 & 0 & 1 & 1 & 0 \\
\hline Total & 218 & 29 & 27 & 27 & 15 & 24 \\
\hline
\end{tabular}

Fuente: elaboración propia con base en los expedientes CNRT 110509/2013 y SOFSE S02:0025646/2016.

Observaciones: la columna "solados hápticos" registra sólo aquellas con los tres tipos de solados, de guía, prevención y peligro, en todos los andenes. Asimismo, las columnas de "rampas", "baños", "ascensores" y "apoyos isquiáticos” registran a aquellas estaciones que cumplen esos requisitos en la totalidad de los andenes. En todos los casos se contabilizan sólo las "rampas" y 
"baños" en los que se ha verificado el respeto a las pautas técnicas establecidas en la Ley 24.314 y su reglamentación.

La información del cuadro 1 coincide con la información brindada en un expediente posterior, del año 2016 (SOFSE S02:0025646). De esta manera, se confirma lo sostenido por el Ceddis y la AGN: ninguna de las líneas de trenes es accesible, ninguna cumple con el marco legal presentado.

A continuación, en el cuadro 2, presentamos la información brindada por la empresa Subterráneos de Buenos Aires S.E., donde se responde al pedido de información sobre la accesibilidad de las distintas estaciones que administra la empresa de subterráneos.

Cuadro 2. Elementos de accesibilidad en las estaciones de las líneas de subterráneos de la Ciudad de Buenos Aires, para el año 2017

\begin{tabular}{|c|c|c|}
\hline Linea & Cantidad de estaciones & Estaciones accesibles \\
\hline A & 18 & 11 \\
\hline B & 17 & 6 \\
\hline C & 9 & 7 \\
\hline D & 16 & 0 \\
\hline E & 15 & 11 \\
\hline H & 11 & 1 \\
\hline
\end{tabular}

Fuente: elaboración propia con base en los expediente SBASE IF-2017-14792616.

Observaciones: se solicitó el nivel de accesibilidad de las distintas estaciones, pero sólo se ha respondido cuál es la cantidad de ascensores en las estaciones de cada línea, omitiendo la información sobre el resto de dispositivos obligatorios.

El cuadro 2 muestra una situación similar a la descripta sobre los trenes de pasajeros en el cuadro 1, aunque con menor información. Sólo se informa sobre la cantidad de ascensores en las estaciones. A pesar de ello, este expediente nos permite confirmar la falta de accesibilidad en los subterráneos de Buenos Aires, como lo ha denunciado Antonieta Aviani a la justicia.

En síntesis, todas las fuentes exponen -en sintonía- que, a pesar de la política pública diseñada por la Ley 22.431 y su decreto reglamentario, la misma no se ha implementado adecuadamente. No se han realizado las obras y acciones necesarias para garantizar la igualdad de oportunidades en el uso del transporte ferroviario por parte del colectivo con discapacidad. Esta situación, que el Poder Judicial había intentado corregir a través de sus sentencias, no fue modificada. 


\section{Conclusiones}

El Poder Judicial se ha ocupado positivamente de la accesibilidad en el transporte ferroviario. Los casos Verbrugghe, Aviani y Defensoría del Pueblo muestran un Poder Judicial comprometido con los derechos humanos, al cumplir su función de balance. Ha ordenado en los tres casos la aplicación efectiva de las políticas públicas que establecen las acciones y medidas para la accesibilidad física en el transporte.

Considerando lo trabajado en nuestra sección tercera, podríamos coincidir con los estudios cuantitativos sobre la calidad de la democracia, como los de Barreda, que encuentran un alto nivel de calidad en la rendición de cuentas. Sin embargo, cuando en la sección cuarta confirmamos que estas sentencias judiciales fueron incumplidas, la perspectiva cambia.

A través del estudio de una política pública concreta para un colectivo específico, como el de discapacidad, y el rol del Poder Judicial de asegurar su cumplimiento, vemos que el componente republicano de la democracia moderna argentina se encuentra debilitado. La mirada más amplia de la poliarquía dahliana que aporta 0'Donnell pone atención en el control al ejercicio del poder político y, particularmente, la rendición de cuentas o accountability.

En este breve trabajo, podemos ver de forma concreta cómo un Poder Judicial que no es totalmente autónomo, pero es respetado en sus decisiones, vulnera el componente liberal de la democracia. Es decir, impide el pleno goce de derechos civiles. Por otra parte, la interdependencia de los tres componentes que plantea el politólogo argentino (democrático, republicano y liberal) permite un análisis más completo e integral para evaluar la calidad de la democracia.

En futuros trabajos incorporaremos a este análisis al resto de las agencias de balance del Estado argentino (Poder Legislativo y Poder Ejecutivo), respecto a su rol y accionar en la política pública de accesibilidad física en el transporte ferroviario. Asimismo, consideramos pertinente que se continúen desarrollando estudios sobre esta temática, sobre todo a nivel subnacional, debido al rol central que tienen las provincias y la Ciudad Autónoma de Buenos Aires en garantizar los derechos humanos del colectivo con discapacidad. 


\section{Bibliografía}

Aвramovich, Víctor, "La situación de los derechos humanos en América Latina”, Panel Derechos Humanos, Autoritarismo y Democracia. Los aprendizajes de la lucha por los derechos humanos para intervenir en los problemas del presente, Buenos Aires, 18 de mayo de 2009.

AlcÁntara, Manuel, "Luces y sombras de la calidad de la democracia de América Latina”, Revista de Derecho Electoral, núm. 6, 2008, pp. 1-15.

Altman, David y PÉrez-LiñÁn, AníBAl, “Assessing the Quality of Democracy: Freedom, Competitiveness and Participation in Eighteen Latin American Countries”, Democratization, núm. 9, 2002, pp. 85-100.

BARRedA, Mikel, "La calidad de la democracia: Un análisis comparado de América Latina”, Politica y gobierno, vol. 18, núm. 2. [Consulta: 12 de marzo, 2017]. Disponible en: http://www.scielo.org.mx/scielo.php?script=sci_arttextEtpi$\mathrm{d}=$ S1665-20372011000200003

BARRedA, Mikel, "La calidad de la accountability de las democracias latinoamericanas”, Politai, vol. 2, núm. 2, 2011. [Consulta: 17 de mayo, 2017]. Disponible en: http://revistas.pucp.edu.pe/index.php/politai/article/view/13936

CEDDIs, "Informe de la República Argentina en virtud del artículo VI.3 de la Convención Interamericana para la eliminación de todas las formas de discriminación contra las personas con discapacidad”, 28 febrero, Panamá, 2007. [Consulta: 19 de mayo, 2017]. Disponible en: http://www.grupodemujeres. org.ar/descargas/vih-prevenir/DISCAPACIDAD\%20-020CEDDIS_doc_807 argentina_esp.doc

DAHL, ROBERT, La poliarquía: participación y oposición, Madrid, Tecnos, 2002.

Fayt, Carlos, El efectivo cumplimiento de las decisiones de la Corte Suprema de Justicia de la Nación. La fuerza imperativa de sus pronunciamientos, Buenos Aires, La Ley, 2007.

Galvagno, Patricia Luján, “Derecho subterráneo”, en Actas de II Jornadas Nacionales “Discapacidad y Derechos”, 29 y 30 de mayo de 2014, Facultad de Derecho, Universidad de Buenos Aires. [Consulta: 12 de marzo, 2017]. Disponible en: http://www.derecho.uba.ar/academica/centro-desarrollo-docente/documentos/segundas-jornadas-nacionales-discapacidad-y-derechos/ eje-1-legislacion-y-jurisprudencia.pdf

Levine, Daniel y Molina, José, "La calidad de la democracia en América Latina: una visión comparada”, América Latina Hoy, núm. 45. [Consulta: 10 de marzo, 2017]. Disponible en: http://revistas.usal.es/index.php/1130-2887/article/ view/2427

Mainwaring, Scott y PÉrez-Liñán, Aníbal, "Regime Legacies and Democratization: Explaining Variance in the Level of Democracy in Latin America, 1978-2004”, documento de trabajo 354, Kellogg Institute for International Studies, 2008. 
Munck, GerARDo, "Repensando la cuestión democrática: la región andina en el nuevo siglo”, Revista de Ciencia Política, vol. 30, núm. 1, 2010.

O’Donnell, Guillermo, "Democracia Delegativa”, Journal of Democracy en español, núm. 1.

O’Donnell, Guillermo, Contrapuntos: estudios escogidos sobre autoritarismo y democratización, Buenos Aires, Paidós, 1997.

O’Donnell, Guillermo, "Why the Rule of Law Matters", Journal of Democracy, vol. 15, núm. 4.

O’Donnell, Gullermo, Disonancias. Críticas a la democracia, Buenos Aires, Paidós, 2007.

Palacios, Agustina, La discapacidad como una cuestión de derechos humanos. Una aproximación a la Convención Internacional sobre los Derechos de las Personas con Discapacidad, Madrid, Ediciones Cinca, 2007.

Pozo Gowland, Héctor, "El incumplimiento de sentencias por el Estado. Ejecución forzosa y poder de sustitución de los jueces, Buenos Aires”, Academia Nacional de Derecho y Ciencias Sociales de Buenos Aires. [Consulta: 19 de junio, 2017]. Disponible en: http://www.pozogowlandabogados.com.ar/pozo-gowland/id/es/notas/_21072011055411/185/

Samaniego, Pilar, Aproximación a la realidad de las personas con discapacidad en Latinoamérica, Madrid, CERMI, 2006.

TAMAYo SÁEz, MANuEl, "El análisis de las políticas públicas”, en Rafael Bañón, Ernesto Carrillo (comps.), La nueva administración pública, Madrid, Alianza, 1997. 
